\title{
Spectral scaling of static pressure fluctuations in the atmospheric surface layer: The interaction between large and small scales
}

\author{
John D. Albertson \\ Department of Environmental Sciences, University of Virginia, Charlottesville, Virginia 22903 \\ Gabriel G. Katul \\ School of the Environment, Duke University, Durham, North Carolina 27708 \\ Marc B. Parlange \\ Department of Geography and Environmental Engineering, The Johns Hopkins University, \\ Baltimore, Maryland 21218 \\ William E. Eichinger \\ Institute of Hydraulic Research, University of Iowa, Iowa City, Iowa 52242
}

(Received 17 March 1997; accepted 2 February 1998)

\begin{abstract}
Static pressure fluctuations measured in the atmospheric surface layer over a grass covered forest clearing are studied in the context of Townsend's 1961 hypothesis regarding the effect of the outer region on the inner region. It is shown that large-scale pressure features are actively straining the inertial-scale pressure fluctuations, thus invalidating the direct extension of Kolmogorov's 1941 hypothesis to the spectral scaling of pressure within the inertial subrange. A parameter describing the large scale pressure fluctuations is added to the set of variables responsible for inertial-range pressure differences and dimensional analysis is employed to derive an improved scaling law for pressure spectra which more closely matches these and previous experimental results. An examination of the Poisson equation for pressure is conducted and found to support the dimensional and experimental results. (C) 1998 American Institute of Physics. [S1070-6631(98)00206-2]
\end{abstract}

\section{INTRODUCTION}

Turbulently fluctuating static pressure is perhaps the least understood basic flow variable in the atmospheric surface layer (ASL). This is due to the difficulty inherent in measuring this term and, consequently, the lack of published experimental results. The static pressure term is intimately linked with the momentum equation. To improve our understanding of momentum dependent processes, such as turbulent transfer between the land and the atmosphere, we require an improved understanding of the pressure behavior. ${ }^{1-3}$ New pressure-sensing instruments have been developed in the past two decades ${ }^{4-6}$ and have been applied in field experiments to yield useful results. However, the spectral properties of the fluctuating static pressure remain largely uncertain.

Following Kolmogorov's ${ }^{7}$ arguments for the inertial subrange, in the case of local isotropy, the $n$th moment of velocity differences $\left(D_{n, u}\right)$ taken over a separation $r$ in the direction of the mean flow is

$$
D_{n, u}=\left\langle(u(x+r)-u(x))^{n}\right\rangle=C_{n, u}\langle\epsilon\rangle^{n / 3} r^{n / 3},
$$

where $u$ is a velocity component, $\epsilon$ is the dissipation rate of turbulent kinetic energy per unit mass, and $\langle\cdots\rangle$ is the averaging operator. The Fourier counterpart of (1) with $n=2$ is the celebrated $k^{-5 / 3}$ scaling law for velocity spectra. It has been suggested that Kolmogorov's similarity arguments in the inertial subrange can be applied to pressure fluctuations as well. ${ }^{8}$ This approach, based predominately on dimensional arguments and the idea that static pressure differences across spatial lag $r$ are described completely by $\epsilon$ and $r$, yields

$$
D_{2, p}=\left\langle(p(x+r)-p(x))^{2}\right\rangle=C_{2, p}\langle\epsilon\rangle^{4 / 3} r^{4 / 3},
$$

where $p$ is the static pressure fluctuation in $\mathrm{m}^{2} / \mathrm{s}^{2}$ (i.e., the pressure is normalized by the density) and $C_{2, p}$ is a constant. We note that (2) can also be derived from theoretical consideration of higher order local velocity moments, ${ }^{9}$ as outlined later in the Discussion. The Fourier counterpart of (2) is the predicted $k^{-7 / 3}$ scaling law for pressure spectra. However, it is not clear whether the Kolmogorov similarity arguments apply to pressure in a wall bounded shear flow. To directly extend Kolmogorov's inertial range assumptions to pressure [as in (2)] is to assume an analogy between the mechanisms that cause pressure differences across spatial separations $(r)$ in the inertial subrange and those mechanisms that cause velocity differences. Such an analogy is useful to explain some of the pressure difference-that part owing to local effects-but it fails to capture the nonlocal contributions to the local pressure field. In fact, Monin and Yaglom ${ }^{8}$ (p. 343) offer these words of caution when describing the usefulness of the local isotropy tools:

"...the application of the theory of locally isotropic turbulence to the study of pressure fluctuations is slightly more questionable than other applications of the theory; it is possible that comparatively far regions of the flow make non-negligible contributions to the pressure fluctuations at a point."'

It is helpful to consider this issue in the context of Townsend's ${ }^{10}$ hypothesis, which describes the turbulent field in the inner region of the atmospheric boundary layer $(\mathrm{ABL})$ 
as the superposition of two fields: one of "active motion" due to the vorticity of the inner region of the boundary layer, and the other of "inactive motion" imposed on the inner region by pressure fluctuations created in the outer region. The active motion is directly due to the local momentum transfer to the surface and is, therefore, governed by the shear stress $\tau$ and the height above the ground $z$. The inactive motion is irrotational and not related to $\tau$. Bradshaw's ${ }^{11}$ data analysis supports Townsend's theory, showing that large scale pressure fluctuations in the outer region affect the dynamics in the inner region. What remains to be investigated is whether these large scale fluctuations are affecting the inertial scale pressure fluctuations. If, in fact, significant interactions exist, then (2) will not accurately describe the local structure of the pressure field. This may explain why (2) has failed to successfully match experimental measurements in the ABL. ${ }^{4,12,13}$

Although several studies of surface static pressure fluctuations have been performed in the laboratory, ${ }^{11,14-18}$ the results are typically scaled by properties not relevant to ASL studies. Consequently, the present discussion is restricted to relevant field studies and some important, yet general, results of the laboratory studies. For a review of the early laboratory studies see Willmarth. ${ }^{19}$

George $e t a l^{20}$ derived from a spectral model of the velocity field an expression for the inertial range pressure spectra for free shear flows. As their work was constrained to free shear flows the surface integral, which accounts for boundary effects, vanished in their integration of the Poisson equation for pressure. The resulting pressure field is shown to be due to two mechanisms: the interaction of turbulence with itself and the interaction of turbulence with the mean shear. This work clarified the scaling of pressure spectra in the inertial subrange for locally isotropic free shear flows, with a finding that it scales as $k^{-7 / 3}$. The focus of the present study is on wall bounded shear flows, where the surface integral cannot be ignored in the Poisson solution. Hence, we can accept the result of George et al. ${ }^{20}$ as an excellent point of departure, from which we can study the added complication of the presence of a wall. An important effect of the wall may well be the imposition of large scale pressure features on the lower region of the boundary layer from perturbations occurring in the outer region. We consider the potential effects of these vertically communicated large scale pressure perturbations on the structure of the inertial scale pressure field in the inner region of the ASL.

Elliott's ${ }^{12}$ work represents the first detailed study of static pressure fluctuations in the free stream flow of the ASL. He measured static pressure spectra in the ASL up to 6 $\mathrm{m}$ above the surface and found no strong dependence on height $z$, unlike inertial range velocity spectra which depend strongly on $z$, and found the spectra to collapse when normalized by the squared shear stress. In both papers Elliott ${ }^{4,12}$ presents pressure spectra that scale with $k$ raised to a power between -1.5 and -1.7 , in stark contrast to the theoretical scaling of $k^{-7 / 3}$ (i.e., slope of -2.333). From measurements below and above a mature forest canopy, Sigmon et al. ${ }^{13}$ found the pressure spectra to be somewhat flatter than $k^{-2}$, with the flattest spectra found above the canopy. They at- tribute the steeper slope in the canopy to the preferential filtering of high frequency fluctuations by the trees. The predominance of low wave number pressure fluctuations inside the canopy is explained well by the analysis of Shaw and Zhang, ${ }^{21}$ which describes how flow inside the canopy is largely driven by large scale pressure perturbations impressed on the lower regions by the flow above the canopy. Analyses of wind tunnel data have shown similar responsibility of the outer region flow in controlling the inner region flow through pressure fluctuations. ${ }^{16,22}$ Interestingly, they found an interdependence between the low- and high-wavenumber pressure fluctuations. This point raises serious doubts about the applicability of local isotropy assumptions in describing the pressure spectrum.

Praskovsky et $a l .{ }^{23}$ have shown that when the large-scale components of a variable are interacting with the "inertial scales" an additional large-scale governing parameter, such as the rms value of the variable, must be added to the inertial-range scaling. ${ }^{22}$ Therefore, assuming that interaction between the large- and small-scales is significant, a measure of the energy content of the large scale static pressure must be considered in the list of dimensional variables influencing the statistical structure of the local pressure.

We seek a scaling form that describes the squared pressure fluctuations across lag $r$ in terms of $\epsilon, r$, and $\sigma_{p}$. In this case there are three important variables and two basic dimensions, length and time. Note that this system is underdetermined in a manner similar to the expression of mean vertical gradients in a boundary layer subject to density stratification. We implement this additional large scale variable by modifying (2) with a new dimensionless group

$$
\frac{D_{2, p}}{\langle\epsilon\rangle^{4 / 3} r^{4 / 3}}=F\left(\frac{r}{\Lambda}\right)
$$

where $\Lambda\left(=\sigma_{p}^{3 / 2} /\langle\epsilon\rangle\right)$, and $F$ is a general similarity function. Although $F$ may, in a general sense, take any form, it must be constrained to a power-law if the resulting expression is to be scale-invariant. This is in keeping with the general concept of an inertial subrange. With $F(r / \Lambda)$ taken as $C_{2, p}^{\prime}\left(\sigma_{p} /\langle\epsilon\rangle^{2 / 3} r^{2 / 3}\right)^{a}$, this new scaling law becomes

$$
D_{2, p}=C_{2, p}^{\prime}\langle\epsilon\rangle^{4 / 3} r^{4 / 3}\left(\frac{\sigma_{p}}{\langle\epsilon\rangle^{2 / 3} r^{2 / 3}}\right)^{a}
$$

where $C_{2, p}^{\prime}$ and the exponent $a$ are new constants to be determined by experiment. Note that (3) converges to (2) when $a \rightarrow 0$. This new form provides a scale-invariant description of the local pressure field in terms of the dissipation rate, the separation distance, and a measure of the influence of the large scale pressure field.

The objectives of the present paper are to (i) evaluate whether significant interaction exists between large- and small-scales over a wide range of conditions in the atmospheric surface layer; (ii) determine if this interaction can explain the consistently flatter-than-predicted spectral scaling slopes observed for the local pressure field in previous boundary layer experiments; and (iii) define for the local 
TABLE I. Summary of selected mean meteorological and turbulence conditions. Runs $1-4$ are DOY $=253$ and runs $5-14$ are $\mathrm{DOY}=254$.

\begin{tabular}{rcccccrrr}
\hline \hline $\begin{array}{c}\text { Run } \\
\#\end{array}$ & $\begin{array}{c}u_{*} \\
\left(\mathrm{~ms}^{-1}\right)\end{array}$ & $\begin{array}{c}H \\
\left(\mathrm{wm}^{-2}\right)\end{array}$ & $\begin{array}{c}L E \\
\left(\mathrm{wm}^{-2}\right)\end{array}$ & $-z / L$ & $\begin{array}{c}\sigma_{p} \\
\left(\mathrm{~m}^{2} \mathrm{~s}^{-2}\right)\end{array}$ & $\begin{array}{c}L_{p} \\
(\mathrm{~m})\end{array}$ & $\begin{array}{r}L_{u} \\
(\mathrm{~m})\end{array}$ & $\begin{array}{c}\langle\epsilon\rangle \times 10^{3} \\
\left(\mathrm{~m}^{2} \mathrm{~s}^{-3}\right)\end{array}$ \\
\hline 1 & 0.20 & 97.0 & 226.0 & 0.25 & 0.07 & 17.2 & 24.5 & 5.90 \\
2 & 0.21 & 87.0 & 211.0 & 0.22 & 0.05 & 14.6 & 23.5 & 7.34 \\
3 & 0.23 & 61.0 & 171.0 & 0.11 & 0.08 & 5.8 & 18.5 & 9.73 \\
4 & 0.20 & 24.0 & 140.0 & 0.08 & 0.06 & 9.6 & 5.5 & 8.25 \\
5 & 0.32 & 99.0 & 134.0 & 0.06 & 0.19 & 53.4 & 72.1 & 24.6 \\
6 & 0.31 & 119.0 & 174.0 & 0.08 & 0.18 & 40.7 & 12.0 & 28.4 \\
7 & 0.29 & 118.0 & 166.0 & 0.10 & 0.14 & 14.0 & 35.2 & 21.9 \\
8 & 0.28 & 134.0 & 208.0 & 0.13 & 0.20 & 22.4 & 39.4 & 27.8 \\
9 & 0.32 & 143.0 & 228.0 & 0.09 & 0.22 & 32.2 & 35.0 & 46.8 \\
10 & 0.23 & 154.0 & 210.0 & 0.27 & 0.14 & 18.0 & 17.1 & 18.6 \\
11 & 0.24 & 112.0 & 208.0 & 0.17 & 0.12 & 58.4 & 35.4 & 19.7 \\
12 & 0.29 & 157.0 & 264.0 & 0.13 & 0.16 & 28.7 & 80.7 & 16.7 \\
13 & 0.16 & 37.0 & 101.0 & 0.21 & 0.06 & 8.5 & 8.5 & 7.41 \\
14 & 0.16 & 47.0 & 113.0 & 0.23 & 0.07 & 17.0 & 16.1 & 5.60 \\
\hline \hline
\end{tabular}

pressure statistical structure in the ASL a general similarity formulation that is consistent with the equations of motion and the results of the previous experiments.

\section{EXPERIMENT}

Near surface atmospheric turbulence was measured on September 10 and 11, 1994 in a grass covered clearing at the Duke University Forest. The clearing is approximately 450 $\mathrm{m}$ by $450 \mathrm{~m}$, within a $13 \mathrm{~m}$ tall stand of pine tress. A threedimensional sonic anemometer (Gill Instruments Ltd., Hampshire, England) was used to measure the three components $(U, V, W)$ of the air velocity and the air temperature $\left(T_{a}\right)$ at $10 \mathrm{~Hz}$. A static pressure probe (described below) was located $0.3 \mathrm{~m}$ to one side of the 3D sonic anemometer and was operated at $10 \mathrm{~Hz}$. A Krypton hygrometer (Campbell Scientific, Logan, UT) was located $0.3 \mathrm{~m}$ to the other side of the 3D sonic anemometer and used to measure turbulent fluctuations in the water vapor concentration $(q)$ in the air at $10 \mathrm{~Hz}$. All three instruments were located at a height of 1.55 $\mathrm{m}$ above the ground, which is covered with grass extending to a height of $0.3 \mathrm{~m}$ (i.e., the instruments were about $1.25 \mathrm{~m}$ above the top of the grass canopy). The data have been divided into 27 files (9 from the first day, 18 from the second), each containing 16384 records and covering a period of 27.3 min (i.e., 16384 records at $10 \mathrm{~Hz}$ ). Of these 27 files collected over the 2 days, eight were disqualified on the basis of excessive nonstationarity due to being collected during early morning or late afternoon hours when the flow was clearly passing from one stability regime to another (e.g., stable to unstable), and five were disqualified for lacking an inertial subrange in the velocity statistics. The presence of an inertial subrange was deduced from examination of the slope of the third order velocity structure function. The remaining 14 files are marked by well defined mean values for each flow variable such that they were readily decomposed into mean and fluctuating components. The instantaneous velocities are represented by $U, V$, and $W$, for the streamwise, spanwise, and vertical directions. Upper case letters are used for the instantaneous values, lower case letters represent fluctuations about the mean, and $\sigma$ is used to represent the standard deviation. The mean micrometeorological and turbulent statistics for the 14 acceptable runs are presented in Table I, where $u_{*}\left(=[\tau / \rho]^{1 / 2}=[-\langle u w\rangle]^{1 / 2}\right)$ is the friction velocity, $\tau$ is the surface shear stress, $\rho$ is the air density, $H$ is the vertical sensible heat flux, $L E$ is the latent heat flux, $-z / L$ is the stability parameter, $L$ is the Obukhov length, $L_{u}$ is the integral length of the longitudinal velocity signal, and $L_{p}$ is the integral length of the pressure signal. The integral length scales were computed from the autocorrelation functions ${ }^{25}$

$$
\begin{aligned}
& L_{u}=\langle U\rangle \int_{0}^{\infty} \frac{\langle u(t) u(t+\theta)\rangle}{\sigma_{u}^{2}} d \theta, \\
& L_{p}=\langle U\rangle \int_{0}^{\infty} \frac{\langle p(t) p(t+\theta)\rangle}{\sigma_{p}^{2}} d \theta,
\end{aligned}
$$

where, in practice, the integration is carried out numerically and stopped at the first zero crossing in the autocorrelation function. The boundary layer stratification for these runs varied from near-neutral to moderately unstable, but should have little effect for inertial range purposes. Note that Taylor's ${ }^{26}$ hypothesis of frozen turbulence is used to convert from time to space. The pressure probe was constructed by Conklin ${ }^{6}$ after the design of Robertson, ${ }^{27}$ tested in a series of wind tunnel experiments, ${ }^{6}$ and found to perform as well as a probe ${ }^{13}$ constructed with the standard design of Elliott. ${ }^{4}$ Conklin's probe samples the pressure through $2 \mathrm{~mm}$ holes on the inside of each of two parallel $15 \mathrm{~cm}$ diam disks, separated by $10 \mathrm{~cm}$. The pressure is measured by a Barocell pressure transducer (Datametrics model 570D-10B-2A1-V1X) with an electronic manometer. An effective low-pass filter was implemented by venting to the atmosphere through a long capillary tube. Wind tunnel tests ${ }^{6}$ show this design to suffer only minor effects for angles of attack of up to $20 \mathrm{deg}$. For additional details on the experiment and the probe see Katul et al. ${ }^{24,28}$ 


\section{RESULTS}

Before accepting (3) as a suitable replacement for (2) we must first establish that (i) an inertial subrange is present for the velocity measurements (and, therefore, should be expected for the pressure), (ii) the local velocity differences are not interacting with local pressure differences, ${ }^{8,29}$ and (iii) there exists significant interaction between the large- and small-scale components of the pressure signal. See Katul et al. $^{29}$ for a discussion of isotropy, anisotropy, component interaction, and the inertial subrange.

In the present analysis we will focus on the structure function form of the spectral scaling, rather than the Fourier power spectra form. The structure function is attractive in that it is more closely tied to Kolmogorov's ${ }^{7}$ original arguments regarding local differences, is not sensitive to the definition of a global mean, does not require modification of the measured data through windowing and tapering, and provides a smoother scaling such that power laws are more visible. Furthermore, the structure function is advantageous in the present effort for it has clear and direct ties to the measures of interaction between large and small scales, as developed by Praskovsky et $a l .{ }^{23}$ and introduced below. For a stationary process the structure function is directly related to the power spectrum by the Fourier transform.

We will use the third order structure function for velocity [i.e., (1) with $n=3$ ] as it is considered a more reliable test of inertial subrange scaling than the second order form. ${ }^{30,31}$ Furthermore, for $n=3$ the constant is known exactly, $C_{3, u}$ $=-4 / 5$, from the work of von Karman and Howarth. ${ }^{32} \mathrm{Be}-$ fore presenting ensemble plots of all 14 runs with the variables nondimensionalized, we examine in detail two sample runs (5 and 11) selected arbitrarily from the group of 14 . In Fig. 1(a) the velocity power spectra are presented and are seen to match well the Kolmogorov ${ }^{7}-5 / 3$ scaling law. The corresponding third order structure functions are shown in Fig. 1(b) alongside the predicted $r^{1}$ scaling from (1). Note that only a narrow window around the inertial subrange is presented in Fig. 1(b) as the third order structure function becomes highly erratic for lags longer than inertial separation as anisotropic effects become significant. The second order structure function is much smoother, asymptotes predictably as $r \rightarrow \infty$, and is, therefore, not as sensitive an indicator of local isotropy in the velocity signal. The third order structure functions were regressed (from $0.15 \mathrm{~m}<r$ $<0.75 \mathrm{~m}$ ) to obtain estimates of $\langle\epsilon\rangle$ for each of the 14 files (see Table I), then normalized by $\langle\epsilon\rangle r$, and plotted against $r / L_{u}$ in Fig. 2. The range regressed is expected to behave inertially as it represents scales between a lower limit set by the instrument's maximum resolution and an upper limit equal to one-half the height above the wall. Note from (1) that $D_{3, u} /(\langle\epsilon\rangle r)$ should have no $r$ dependence in the inertial subrange. It appears from Fig. 2 that the velocity signals scale in accordance with Kolmogorov's ${ }^{7}$ theory, thus meeting an important, necessary, but not sufficient condition for the presence of an inertial subrange.

The interaction of streamwise velocity differences $\Delta u(r)(=u(x+r)-u(x))$ and pressure differences $\Delta p(r)(=p(x+r)-p(x))$ across lag $r$ in the inertial sub-
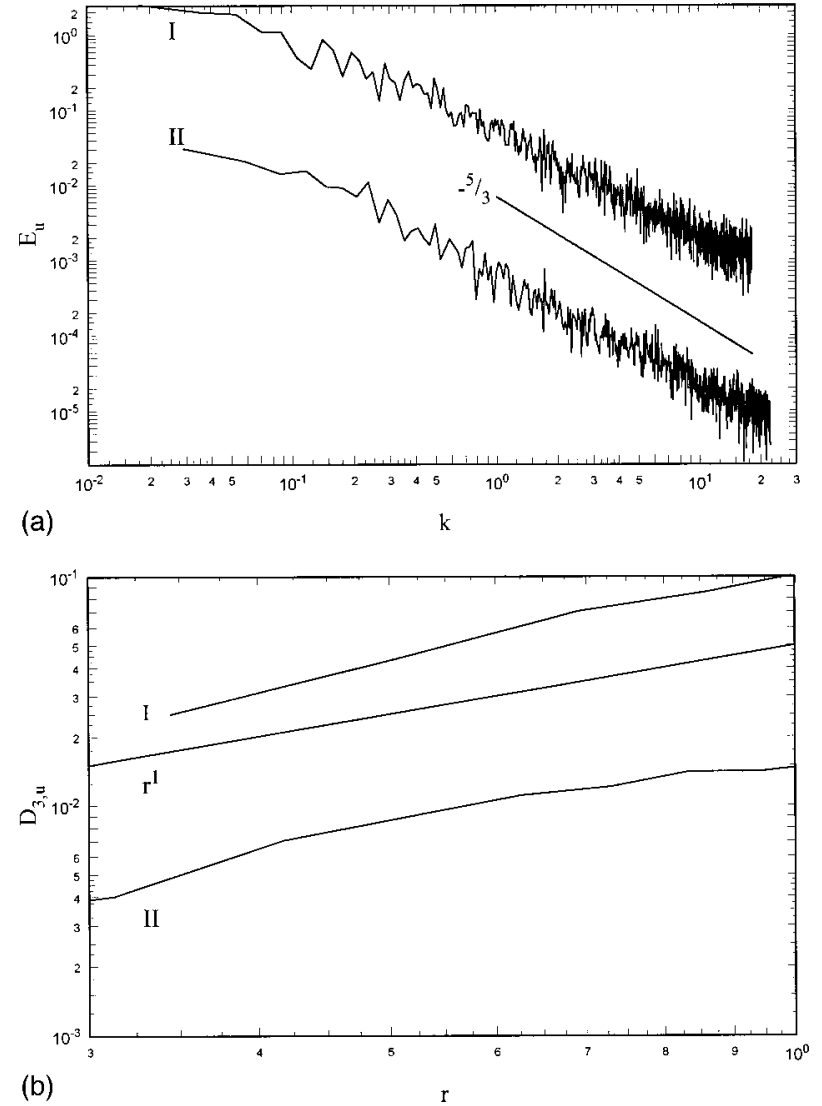

FIG. 1. (a) Longitudinal velocity power spectra for two sample files. The Kolmogorov $-5 / 3$ spectrum is shown for comparison. The wave number $k$ has units of $(\mathrm{rad} / \mathrm{m})$. (b) Third order structure function of the longitudinal velocity for the same two files shown in (a). The predicted inertial range scaling of $r^{1}$ is shown. The lag $r$ is in units of (m).

range was investigated by studying the correlation coefficient between $\Delta u(r)$ and $\Delta p(r)$ as a function of $r$. The correlation coefficient between $\Delta u(r)$ and $\Delta p(r)$ is shown in Fig. 3 for a wider range of $r$ than in Fig. 2 in order to depict the contamination of $\Delta p(r)$ by $\Delta u(r)$ as $r$ becomes larger than

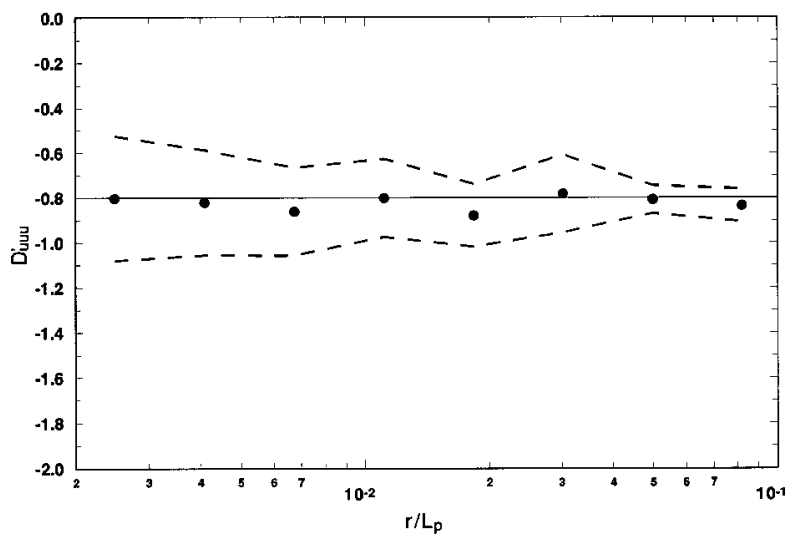

FIG. 2. Ensemble average (14 files) of the normalized third order structure function of the longitudinal velocity (solid circles), plotted with \pm one standard deviation about the ensemble average (dashed lines). The normalized abscissa, $D_{\text {uuu }}^{\prime}(r)=D_{3, u}(r)\langle\epsilon\rangle^{-1} r^{-1}$, should be constant $(-4 / 5)$ for lags $r$ in the inertial subrange. The ordinate has been normalized by the integral length scale of the longitudinal velocity. 


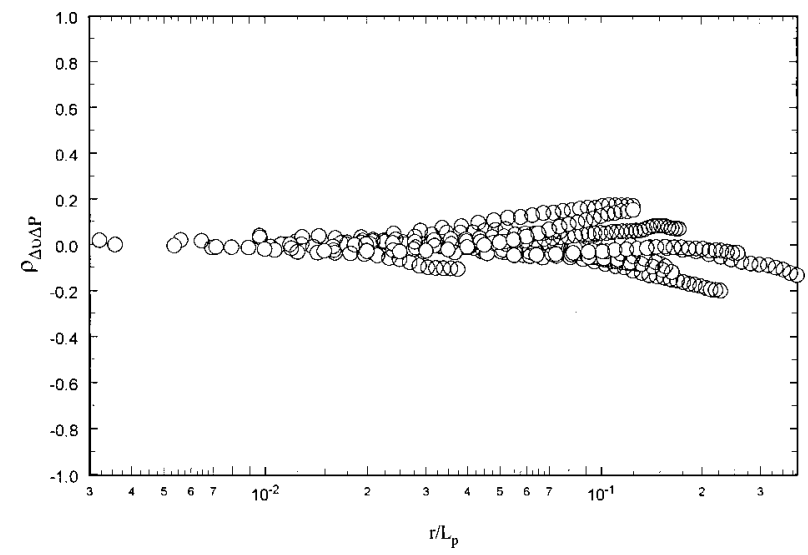

FIG. 3. The correlation coefficient between $\Delta p(r)$ and $\Delta u(r)$ plotted against lag normalized by the integral length scale of pressure.

the measurement height. From Fig. 3 it is evident that for the assumed inertial scales there is a near absence of interaction of $\Delta p(r)$ and $\Delta u(r)$, consistent with local isotropy conditions as described by Monin and Yaglom ${ }^{8}$ (pp. 401-402).

To examine the interaction of large- and small-scale pressure we employed a correlation coefficient between large scale pressure fluctuations $p$ and squared local pressure differences $\Delta^{2} p(r)\left[=(p(x+r)-p(x))^{2}\right]$ as introduced by Praskovsky et al. ${ }^{23}$

$$
\rho_{p \Delta p^{2}}=\frac{\left\langle p(x) \cdot\left(\Delta^{2} p(r)-\left\langle\Delta^{2} p(r)\right\rangle\right)\right\rangle}{\sigma_{p} \sigma_{\Delta_{p}^{2}}}
$$

and found the correlation to be consistently of the order $20 \%-30 \%$ throughout the inertial subrange. This is an extension of the arguments (about velocity) made by Praskovsky et al. (1993), resulting in the selection of $p$ and $\Delta p(r)$ to represent "global" and "local" pressure scales. The presence of finite correlation between these flow variables implies dynamical interaction between them.

From the above analysis we have reason to expect that the flow possesses an inertial subrange, that local velocity differences are not interacting with local pressure differences, and that the large scale pressure is actively straining the local pressure differences. To account for the effect of the large scale pressure, we add $\sigma_{p}$ to the list of important dimensional variables that describe the scaling of the local pressure field. Here $\sigma_{p}$ is used as a proxy for the energy content of the large scale pressure fluctuations.

We now explore the values of $C_{2, p}^{\prime}$ and $a$ in (3). As with the velocity, we first show the power spectra and structure functions for runs 5 and 11. Figure 4(a) depicts the power spectra of the pressure fluctuations. Note that rather than scaling as $k^{-7 / 3}$ they seem to be scaling as $k^{-3 / 2}$, as seen in previous field experiments. ${ }^{4,12,13}$ The second order pressure structure function is shown in Fig. 4(b), where we note the inertial scales following $r^{1 / 2}$ rather than $r^{4 / 3}$. Note $r^{1 / 2}$ and $r^{4 / 3}$ correspond directly to $k^{-3 / 2}$ and $k^{-7 / 3}$, respectively, and that in Fig. 4(b) we are able to present a wider range of $r$ than in Fig. 1(b) because the second order statistics are less prone to erratic behavior from sign changes as $r$ gets larger than the inertial scales. With $\langle\epsilon\rangle$ known from the regression

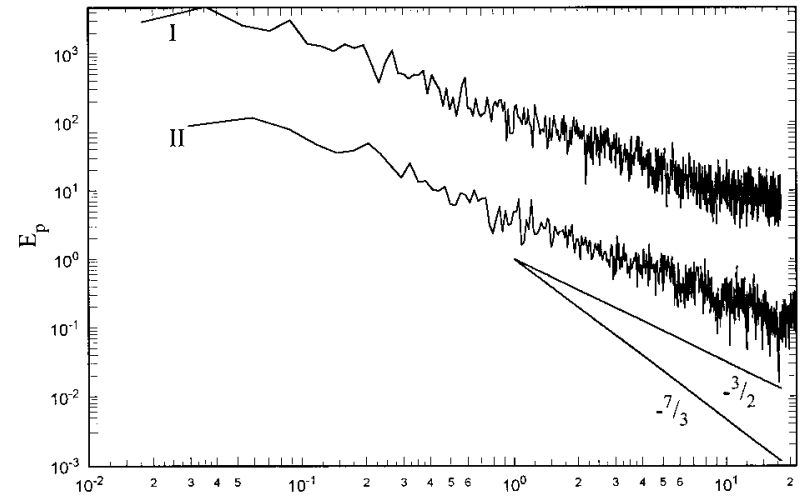

(a)

$\mathrm{k}$

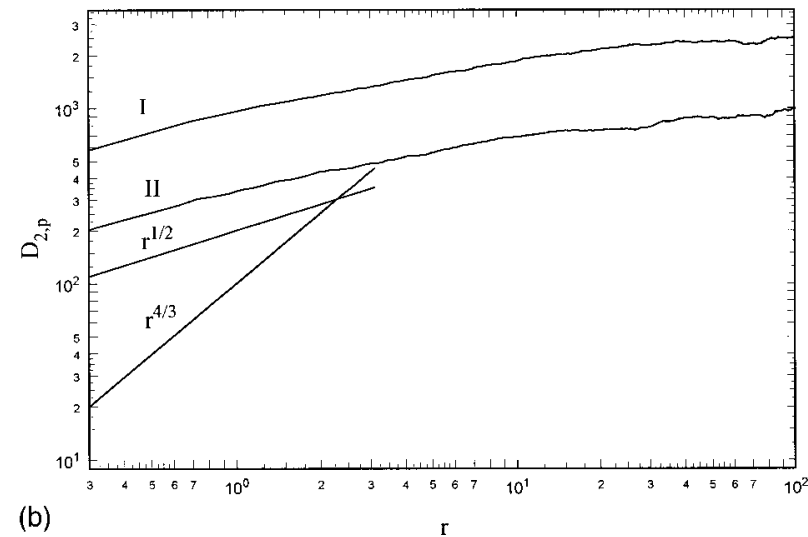

FIG. 4. (a) Power spectra of pressure for the two sample files. The $-7 / 3$ and $-3 / 2$ scaling forms are shown for comparison. The wave number $k$ has units of $(\mathrm{rad} / \mathrm{m})$. (b) Second order structure function of pressure for the two sample files. The two predicted inertial range scaling forms of $r^{4 / 3}$ and $r^{1 / 2}$ are shown. The lag $r$ has units of $(\mathrm{m})$.

of $D_{3, u}$, (3) was log transformed and subject to regression to obtain estimates of $C_{2, p}^{\prime}$ and $a$ for each data file (Table II). From an inspection of Table II, it seems reasonable to assign a value of unity to $C_{2, p}^{\prime}$, which for the 14 files has a mean value of 0.97 and a standard deviation of 0.17 , and a value of $5 / 4$ for $a$, which for the 14 values has a mean of 1.27 and a standard deviation of 0.06 . Therefore (3) becomes

TABLE II. Regressed constants for Eq. (3). Note that the net "power" on $r=4 / 3-2 a / 3$.

\begin{tabular}{rccc}
\hline \hline File & $C_{2, p}^{\prime}$ & $a$ & Power \\
\hline 1 & 1.08 & 1.22 & 0.52 \\
2 & 0.76 & 1.25 & 0.50 \\
3 & 0.98 & 1.28 & 0.48 \\
4 & 0.68 & 1.13 & 0.58 \\
5 & 1.18 & 1.34 & 0.44 \\
6 & 0.95 & 1.27 & 0.49 \\
7 & 1.31 & 1.27 & 0.49 \\
8 & 0.85 & 1.28 & 0.48 \\
9 & 0.94 & 1.34 & 0.44 \\
10 & 1.12 & 1.36 & 0.43 \\
11 & 0.80 & 1.25 & 0.50 \\
12 & 1.00 & 1.28 & 0.48 \\
13 & 0.83 & 1.21 & 0.53 \\
14 & 0.83 & 1.21 & 0.53 \\
\hline \hline
\end{tabular}




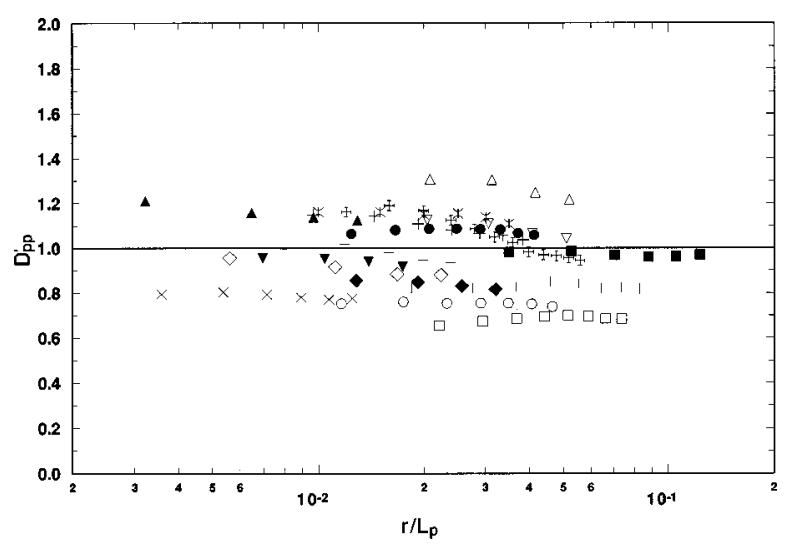

FIG. 5. Normalized second order structure function of pressure for all files. Each file has a unique symbol type. The normalized abscissa, $D_{p p}^{\prime}(r)$ $=D_{2, p}(r)\left[\sigma_{p}^{5 / 4}\langle\epsilon\rangle^{1 / 2} r^{1 / 2}\right]^{-1}$, should be constant for lags $r$ in the inertial subrange. The ordinate has been normalized by the integral length scale of pressure.

$$
D_{2, p}=\left\langle(p(x+r)-p(x))^{2}\right\rangle=C_{2, p}^{\prime} \sigma_{p}^{5 / 4}\langle\epsilon\rangle^{1 / 2} r^{1 / 2},
$$

where we take $C_{2, p}^{\prime}=1$ from the present experiment. The second order pressure structure functions for the 14 files $(0.15 \mathrm{~m}<r<0.75 \mathrm{~m})$ normalized by $\sigma_{p}^{5 / 4}\langle\epsilon\rangle^{1 / 2} r^{1 / 2}$ are plotted against $r / L_{p}$ in Fig. 5. Note from (7) that $D_{2, p} /\left(\sigma_{p}^{5 / 4}\langle\epsilon\rangle^{1 / 2} r^{1 / 2}\right)$ should have no $r$ dependence in the inertial subrange, as is confirmed by Fig. 5 .

\section{DISCUSSION}

The addition of $\sigma_{p}$ to the list of parameters governing pressure differences over inertial separations was justified by the finite and significant correlation between $p$ and $\Delta^{2} p(r)$. By adding an additional parameter the dimensional analysis yields a scaling form that is variable in the power of $r$, viz., (3). It was necessary to introduce experimental evidence in order to select a specific form of the scaling (7). However, the significance of this result may be clarified by considering the resulting scaling form of $D_{2, p}$ in the context of the equations of motion.

For an incompressible constant density fluid, the momentum and continuity equations are

$$
\begin{aligned}
& \partial_{0} U_{\alpha}+U_{\beta} \partial_{\beta} U_{\alpha}=-\partial_{\alpha} P+\nu \partial_{\beta} \partial_{\beta} U_{\alpha}-\delta_{\alpha 3} g, \\
& \partial_{\alpha} U_{\alpha}=0,
\end{aligned}
$$

where $g$ is the gravitational acceleration, $\nu$ is the kinematic viscosity, $\delta_{\alpha \beta}$ is Kronecker's delta, and for compactness we write $\partial_{0}$ for the local time derivative, $\partial_{\gamma}$ for the partial derivative with respect to the $x_{\gamma}$ direction, and use Einstein's summation notation for terms with repeated subscripts. An equation for the pressure field is obtained by taking the divergence of (8) and enforcing continuity

$$
\partial_{\alpha} \partial_{\alpha} P=-\partial_{\alpha} \partial_{\beta}\left(U_{\alpha} U_{\beta}\right) \text {. }
$$

It is clear from (10) that the pressure field at a point depends on an integration over the full velocity field, including appropriate boundary conditions. An expression for the pressure structure function can be obtained from (10) by writing a similar equation for an adjacent point (separated by dis- tance $r$ ), subtracting (10) from the new equation, taking the expectation of the squared difference, invoking an assumption of local isotropy, and applying Millionschikov's zerofourth cumulant hypothesis ${ }^{8}$ (p. 407) to obtain

$$
\nabla^{2} D_{2, p}=d_{r}^{4} D_{2, p}(r)+\frac{4}{r} d_{r}^{3} D_{2, p}(r)=f(r),
$$

where $f(r)$, for locally isotropic turbulence, is a function of $D_{2, u}, d_{r} D_{2, u}, d_{r}^{2} D_{2, u}$, and $d_{r}^{3} D_{2, u}$. Such an analysis results in

$$
f(r)=-C\langle\epsilon\rangle^{4 / 3} r^{-8 / 3},
$$

where $C$ is a constant related to $C_{2, u}$. We wish to depart from the typical solution, which uses (12) in the solution of (11), and investigate a more general region of solution space to learn about the compatibility of (3) with the equations of motion.

If the statistical structure of the local pressure field is solely a function of $r$, then the Laplacian $\Delta^{2}\left(=d_{r}^{4}\right.$ $\left.+(4 / r) d_{r}^{3}\right)$ will not be altered from the original derivation, and the solution to the homogeneous ordinary differential equation $\left(\nabla^{2} D_{2, p}=0\right)$ must remain the same. Furthermore, the homogeneous solution, subject to the conditions that $D_{2, p}$ remain finite as $r \rightarrow \infty$ and that $D_{2, p} \rightarrow 0$ as $r \rightarrow 0$, results in a trivial solution $D_{2, p}=0$. This dictates that the interaction between the pressure scales must arise in the nonhomogeneous term $f(r)$. Since the earlier experiments suggest a constant slope for the pressure spectrum in a log-log framework, and the homogeneous solution does not yield a power law, we deduce that $f(r)$ is a general function of $r$ with a power law form. Such an assumption requires that the cross-scale interaction not result in strong departures from the locally isotropic state. This approach is analogous to Kolmogorov' ${ }^{33}$ 1962 hypothesis, which introduced a large-scale length parameter in the description of $\Delta u$, and is in keeping with the random sweeping decorrelation hypothesis. Consequently, we study the linear fourth order ordinary differential equation

$$
d_{r}^{4} D_{2, p}(r)+\frac{4}{r} d_{r}^{3} D_{2, p}(r)=A r^{B},
$$

where $A$ contains whatever dimensional parameters are necessary (in addition to $r^{B}$ ) to describe $D_{2, p}$. By change of variable, (13) is reduced to a first order linear ODE that is solved with an integrating factor. Then through successive integration back to the original variable we find the solution

$$
\begin{aligned}
D_{2, p}(r)= & \frac{A r^{4+B}}{(5+B)(4+B)(3+B)(2+B)}+\frac{A_{1}}{6 r}+\frac{A_{2} r^{2}}{2} \\
& +A_{3} r+A_{4} .
\end{aligned}
$$

Upon applying the boundary conditions ${ }^{8}$ (p. 408),

$$
D_{2, p}(0)=0 ; \quad d_{r} D_{2, p}(0)=0 ; \quad \frac{D_{2, p}(r)}{r^{2}} \rightarrow 0, \quad \text { for } r \rightarrow \infty,
$$

the solution (14) becomes 


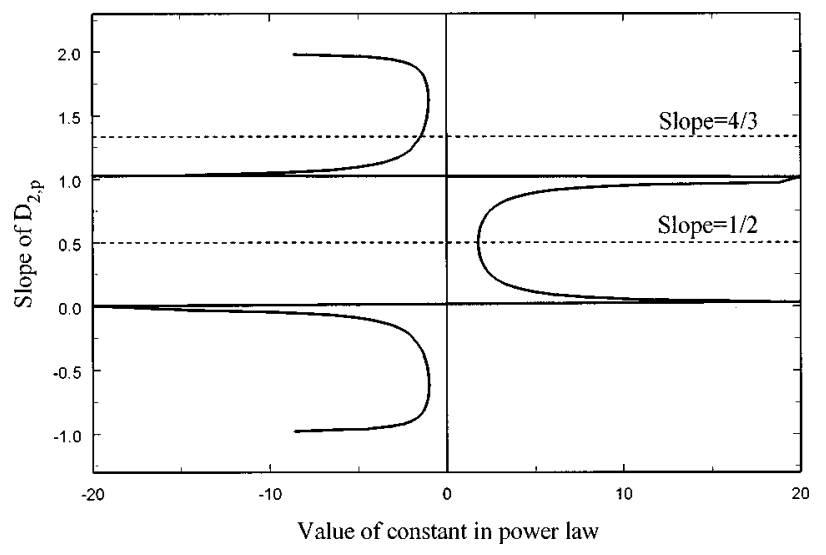

FIG. 6. Relationship between inertial range scaling slope for second order structure function of pressure and value of the constant derived from analysis of the Poisson equation for pressure. Note that the "constant" remains constant for only select, narrow ranges of the slope.

$$
\begin{aligned}
D_{2, p}(r)= & \frac{A r^{4+B}}{(5+B)(4+B)(3+B)(2+B)}, \\
& \text { where } B \leqslant-2 .
\end{aligned}
$$

Note that this solution is singular for integer values of $B$ between -2 and -5 . It is interesting that the constant in front of this scaling form for $D_{2, p}$ is a function of $B$. We expect that $B$ should assume a value about which slight variations in the slope produce at most small variations in the constant. Furthermore, we expect the value of $B$ to be between two limits; the Kolmogorov inertial range limit of $(-8 / 3)$, and the production range (low wave number) limit ${ }^{26}$ of -4 . In keeping with the general concept of an inertial subrange, the quantity $((5+B)(4+B)(3+B)(2+B))^{-1}$ should be a constant. To ensure the maximum possible universality, we propose to define $B$ such that the quantity $((5+B)(4+B)(3+B)(2+B))^{-1}$, in the range $(-8 / 3)$ $<B<-4$, is least sensitive to slight changes in the slope. We study this quantity as a function of slope $(4+B)$ in Fig. 6 . The derivative of this quantity with respect to $B$ possesses one root at $B=-3.5$. This value of $B$ suggests a scaling for $D_{2, p}(r)$ of the form

$$
D_{2, p}(r)=A_{5} A r^{1 / 2} \text {, }
$$

where $A_{5}$ is a constant to be determined from experiment, and $A$ is a dimensional group that contains important parameters to describe $D_{2, p}(r)$ and to achieve dimensional homogeneity. As our objective is to account for nonlocal effects in addition to the local effects described by the Kolmogorov similarity analogy, we admit $\sigma_{p}$ to account for the nonlocal effects and retain $\langle\epsilon\rangle$. Therefore, we replace $A$ with $\left(\langle\epsilon\rangle^{\alpha_{1}} \sigma_{p}^{\alpha_{2}}\right)$ and force dimensional homogeneity on (17) to obtain the scaling form

$$
D_{2, p}(r)=A_{5} \sigma_{p}^{5 / 4}\langle\epsilon\rangle^{1 / 2} r^{1 / 2}
$$

While it is not a rigorous proof, it remains encouraging that this exploration of the Poisson equation results in a scaling form that is consistent with our dimensional analysis. We conclude that (7) is more representative of surface layer pressure fluctuations than the scaling obtained by direct exten- sion of Kolmogorov's ${ }^{7}$ arguments to pressure [as in (2)]. The derivation from (11) to (17) suggests that local isotropy is necessary for (7) to be universal.

In summary, from a review of the salient literature we have noted that (i) pressure spectra in the ASL have, as a rule, not matched the scaling suggested by a straight extension of Kolmogorov's ${ }^{7}$ arguments to static pressure; (ii) inner region flow is affected by low frequency pressure fluctuations in the outer region; and (iii) an interdependence of low- and high-frequency pressure fluctuations has been documented in laboratory experiments. These points motivated an experimental investigation of ASL pressure fluctuations. The resulting data were studied and found to possess an inertial subrange for the velocity components, suggesting that the eddy motion is locally isotropic. No significant interaction was found between velocity differences and pressure differences for spatial separations in the inertial subrange. However, following the approach of Praskovsky et al. ${ }^{23}$ significant interaction was found between large-and small-scale components of the pressure signal. This interaction requires the inclusion of a large-scale pressure parameter $\left(\sigma_{p}\right)$ to the inertial-range scaling of pressure, which resulted in a general spectral scaling form for pressure fluctuations that more accurately accounts for actual boundary layer dynamics. The experimental data are in agreement with the new scaling form and supported the determination of the empirical constants. An examination of the equations of motion also supports the empirical results, leading us to conclude that the second order pressure structure function scales with $r^{1 / 2}$ and the Fourier pressure spectrum scales with $k^{-3 / 2}$ for flows in the surface layer of the atmospheric boundary layer.

\section{ACKNOWLEDGMENTS}

The authors would like to thank Judd Edeburn and Cheng-I Hsieh for their assistance at the Duke Forest, and three anonymous reviewers for their helpful comments. This research has been supported and financed, in part, by the NASA Graduate Student Fellowship in the Global Change Research program, the Environmental Protection Agency (91-0074-94; CR817766), the National Science Foundation (EAR-93-04331 and ATM-7825944), CA State Salinity/ Drainage Task Force, Kearney Foundation, and the UC Davis Superfund grant (5 P42ES04699-07).

${ }^{1}$ G. A. McBean and J. A. Elliott, "The vertical transports of kinetic energy by turbulence and pressure in the boundary layer,"' J. Atmos. Sci. 32, 753 (1975).

${ }^{2}$ J. M. Wilczak and J. A. Businger, "Large-scale eddies in the unstably stratified atmospheric surface layer. Part II: Turbulent pressure fluctuations and the budgets of heat flux, stress and turbulent kinetic energy,' J. Atmos. Sci. 41, 3551 (1984).

${ }^{3}$ J. L. J. Schols and L. Wartena, "A dynamical description of turbulent structures in the near neutral atmospheric surface layer: The role of static pressure fluctuations," Boundary-Layer Meteorol. 34, 1 (1986).

${ }^{4}$ J. A. Elliott, "Instrumentation for measuring static pressure fluctuations within the atmospheric boundary layer," Boundary-Layer Meteorol. 2, 476 (1972a).

${ }^{5}$ R. T. Nishiyama and A. J. Bedard, Jr., “A 'Quad-Disc' static pressure probe for measurement in adverse atmospheres: With a comparative review of static pressure probe designs," Rev. Sci. Instrum. 62, 2193 (1991). 
${ }^{6} \mathrm{P}$. S. Conklin, "Turbulent wind temperature and pressure in a mature hardwood canopy," Ph.D. thesis, Department of Environmental Studies, Duke University, Durham, North Carolina, 1994.

${ }^{7}$ A. N. Kolmogorov, "The local structure of turbulence in incompressible viscous fluid for very large Reynolds numbers," Dokl. Akad. Nauk SSSR 30 [reprinted in Proc. R. Soc. London, Ser. A 434, 9 (1941)].

${ }^{8}$ A. S. Monin and A. M. Yaglom, Statistical Fluid Mechanics (MIT Press, Cambridge, 1975), Vol. 2, p. 874.

${ }^{9}$ R. J. Hill and J. M. Wilczak, "Pressure structure functions and spectra for locally isotropic turbulence," J. Fluid Mech. 296, 247 (1995).

${ }^{10}$ A. A. Townsend, "Equilibrium layers and wall turbulence," J. Fluid Mech. 11, 97 (1961).

${ }^{11} \mathrm{P}$. Bradshaw, " 'Inactive' motion and pressure fluctuations in turbulent boundary layers," J. Fluid Mech. 30, 241 (1967).

${ }^{12} \mathrm{~J}$. A. Elliott, "Microscale pressure fluctuations measured within the lower atmospheric boundary layer," J. Fluid Mech. 53, 351 (1972).

${ }^{13}$ J. T. Sigmon, K. R. Knoerr, and E. J. Shaughnessy, "Microscale pressure fluctuations in a mature deciduous forest," Boundary-Layer Meteorol. 27, 345 (1983)

${ }^{14} \mathrm{~W}$. W. Willmarth and C. E. Woolridge, "Measurements of the fluctuating pressure at the wall beneath a thick turbulent boundary layer," J. Fluid Mech. 14, 187 (1962).

${ }^{15} \mathrm{~W}$. K. Blake, "Turbulent boundary-layer wall-pressure fluctuations on smooth and rough walls," J. Fluid Mech. 44, 637 (1970).

${ }^{16}$ A. S. W. Thomas and M. K. Bull, "On the role of wall-pressure fluctuations in deterministic motions in the turbulent boundary layer," J. Fluid Mech. 128, 283 (1983).

${ }^{17}$ W. L. Keith and J. C. Bennett, Jr., "Low-frequency spectra of the wall shear stress and wall pressure in a turbulent boundary layer," AIAA J. 29, 526 (1991)

${ }^{18}$ W. L. Keith, D. A. Hurdis, and B. M. Abraham, "A comparison of turbulent boundary layer wall-pressure spectra," Trans. ASME 114, 338 (1992).

${ }^{19}$ W. W. Willmarth, "Pressure fluctuations beneath turbulent boundary layers,' Annu. Rev. Fluid Mech. 7, 13 (1975).

${ }^{20}$ W. K. George, P. D. Beuther, and R. E. A. Arndt, "Pressure spectra in turbulent free shear flows," J. Fluid Mech. 148, 155 (1984).

${ }^{21}$ R. H. Shaw and X. J. Zhang, "Evidence of pressure-forced turbulent flow in a forest,"' Boundary-Layer Meteorol. 58, 273 (1992).

${ }^{22}$ S. R. Snarski and R. M. Lueptow, "Wall pressure and coherent structures in a turbulent boundary layer on a cylinder in axial flow," J. Fluid Mech. 286, 137 (1995).

${ }^{23}$ A. A. Praskovsky, E. B. Gledzer, M. Y. Karyakin, and Y. Zhou, "The sweeping decorrelation hypothesis and energy-inertial scale interaction in high Reynolds number flows," J. Fluid Mech. 248, 493 (1993).

${ }^{24}$ G. G. Katul, M. B. Parlange, J. D. Albertson, and C.-R. Chu, "An investigation of the sweeping decorrelation hypothesis in atmospheric surface layer flows," Fluid Dyn. Res. 16, 275 (1995).

${ }^{25}$ J. C. Kaimal and J. J. Finnigan, Atmospheric Boundary Layer Flows (Oxford University Press, Oxford, 1994), p. 289.

${ }^{26}$ G. I. Taylor, "The spectrum of turbulence,' Proc. R. Soc. A 164, 476 (1938).

${ }^{27}$ P. Robertson, “A direction-insensitive static head sensor," J. Phys. E 5, 1080 (1972).

${ }^{28}$ G. G. Katul, J. D. Albertson, C.-I. Hsieh, P. S. Conklin, J. T. Sigmon, M. B. Parlange, and K. R. Knoerr, "The 'inactive' eddy-motion and the largescale turbulent static pressure fluctuations in the dynamic sublayer," J. Atmos. Sci. 53, 2512 (1996).

${ }^{29}$ G. G. Katul, C. I. Hsieh, and J. Sigmon, "Energy-inertial scale interaction for temperature and velocity in the unstable surface layer," BoundaryLayer Meteorol. 82, 49 (1997).

${ }^{30}$ F. Anselmet, Y. Gagne, E. J. Hopfinger, and R. A. Antonia, "High-order velocity structure functions in turbulent shear flows," J. Fluid Mech. 140, 63 (1984).

${ }^{31}$ J. D. Albertson, M. B. Parlange, G. Kiely, and W. E. Eichinger, "The average dissipation rate of turbulent kinetic energy in the neutral and unstable atmospheric surface layer,' J. Geophys. Res. 102, 13423 (1997).

${ }^{32}$ T. von Karman and L. Howarth, "On the statistical theory of isotropic turbulence,'” Proc. R. Soc. London, Ser. A 164, 192 (1938).

${ }^{33} \mathrm{~A}$. N. Kolmogorov, "A refinement of previous hypotheses concerning the local structure of turbulence in a viscous incompressible fluid at high Reynolds number,' J. Fluid Mech. 13, 82 (1962). 